\title{
Cisplatin promotes mesenchymal-like characteristics in osteosarcoma through Snail
}

\author{
SHUO FANG ${ }^{1}$, LING YU $^{1}$, HONGJUN MEI ${ }^{2}$, JIAN YANG ${ }^{1}$, TIAN GAO ${ }^{3}$, \\ ANYUAN CHENG ${ }^{1}$, WEICHUN GUO ${ }^{1}$, KEZHOU XIA ${ }^{1}$ and GAIWEI LIU ${ }^{1}$ \\ ${ }^{1}$ Department of Orthopedics, Renmin Hospital of Wuhan University, Wuhan, Hubei 430060; \\ ${ }^{2}$ Department of Orthopedics, Wuhan No. 5 Hospital, Wuhan, Hubei 430050; ${ }^{3}$ Department of \\ Orthopedic Oncology, Key Laboratory of Carcinogenesis and Translational Research, \\ Ministry of Education, Peking University Cancer Hospital and Institute, Beijing 100871, P.R. China
}

Received June 25, 2015; Accepted October 11, 2016

DOI: $10.3892 / \mathrm{ol} .2016 .5342$

\begin{abstract}
More than $30 \%$ of patients with osteosarcoma succumb to pulmonary metastases. Epithelial-mesenchymal transition (EMT) is a biological process by which tumor cells gain an increased capacity for invasiveness and metastasis. A previous study confirmed the phenomenon of EMT in osteosarcoma, a mesenchymal-derived tumor. However, whether chemotherapy affects EMT remains to be elucidated. In the present study, the osteosarcoma cells were exposed to a sublethal dose of cisplatin, and any surviving cells were assumed to be more resistant to cisplatin. In addition, these cells exhibited a more mesenchymal phenotype. Immunofluorescence analysis revealed that the cisplatin treated cells had an increased long/short axis ratio and increased expression of $\mathrm{N}$-cadherin compared with control cells. A panel of EMT-associated genes was subsequently assessed by quantitative PCR and western blot analysis, and they were observed to be significantly upregulated in the cisplatin treated cells. The in vitro wound healing and Transwell assay indicated that the cisplatin treated cells were more prone to migrate and invade. An in vivo assay showed that the cisplatin-treated xenograft had increased expression of EMT-associated genes, and exhibited increased pulmonary lesions compared with the control, which indicated an elevated capacity to metastasize. The expression of Snail was knocked down by specific small interfering RNA, and it was observed that Snail inhibition promoted cisplatin sensitivity, and cisplatin-induced EMT was significantly blocked. Taken together, the results of the present study supported that idea that Snail participates in cisplatin-induced EMT in osteosarcoma cells, and targeting
\end{abstract}

Correspondence to: Professor Weichun Guo, Department of Orthopedics, Renmin Hospital of Wuhan University, 238 Jiefang Road, Wuhan, Hubei 430060, P.R. China

E-mail: guoweichun@aliyun.com

Key words: osteosarcoma, cisplatin, epithelial-mesenchymal transition, snail, targeted therapy
EMT-transcription factors may offer promise for the therapeutics of osteosarcoma.

\section{Introduction}

Osteosarcoma is the most common primary bone malignancy. It is derived from primitive bone-forming mesenchymal cells and frequently arises in the metaphyses of long bones $(1,2)$. The introduction of chemotherapy significantly improves the outcome for patients, and 5-year event-free survival for localized osteosarcoma increases from $<20$ to $60-65 \%(2,3)$. However, the overall 5-year survival rate for osteosarcoma remains unchanged and has exhibited no marked improvement over recent decades (2).

Cisplatin is one of the most widely used and effective chemotherapy drugs for the treatment of various solid tumors, including those of the breast, brain, lung and testis (4-7). It is an alkylating agent and works by causing DNA lesions via the formation of intrastrand and interstrand crosslinks (8). Cisplatin has been widely used in the treatment of osteosarcoma (9). However, chemoresistance and pulmonary metastasis frequently lead to treatment failure, and the underlying mechanisms remain to be fully elucidated.

The epithelial-mesenchymal transition(EMT) is a biological process by which epithelial cells lose their polarity, disassemble the cell-cell adhesion and become mesenchymal-like cells (10). The process of EMT is accompanied by a reduction in the cell-cell adhesion molecule E-cadherin, and upregulation of more plastic mesenchymal proteins, including vimentin, $\mathrm{N}$-cadherin and smooth muscle actin $(11,12)$. A number of transcription factors (TFs), including Snail/Slug and zinc finger E-box binding homeobox (Zeb)1/2, are involved in this process. EMT allows the tumor cells to gain elevated migratory properties and increased invasiveness, which is a critical step in the process of metastasis leading to cancer spreading and treatment failure $(13,14)$. Chemotherapy has been reported to induce EMT in tumor cells. A previous study by the present authors revealed that osteosarcoma cells expressed a number of EMT-associated genes, which implied that EMT also has a role in mesenchymal-derived sarcoma (15). However, whether cisplatin induces EMT in osteosarcoma remains to 
be elucidated. In the present study, the shape of U2OS cells tended to be diamond and they exhibited an epithelial phenotype compared with other osteosarcoma cell lines. Therefore, this cell line was selected to investigate the process of EMT in osteosarcoma.

In the present study, it was observed that cisplatin exposure promoted mesenchymal characteristics in osteosarcoma and the underlying mechanisms involved upregulation of Snail. These data may provide scientific information for targeted therapy of osteosarcoma.

\section{Materials and methods}

Cell culture. The human osteosarcoma cell line U2OS was obtained from the China Center for Type Culture Collection (Wuhan, China). U2OS cells were cultured in Dulbecco's modified Eagle's medium (DMEM; Thermo Fisher Scientific, Inc., Waltham, MA, USA) containing $10 \%$ (v/v) fetal bovine serum (FBS; Thermo Fisher Scientific, Inc.) and 1\% (v/v) antibiotics $\left(10^{5} \mathrm{U} / \mathrm{ml}\right.$ penicillin, $10^{5} \mu \mathrm{g} / \mathrm{ml}$ streptomycin; GE Healthcare Life Sciences, Logan, UT, USA). Cells were propagated in a humidified environment at $37^{\circ} \mathrm{C}$ with $5 \% \mathrm{CO}_{2}$ and $100 \%$ humidity. Cell viability was determined using trypan blue staining (Thermo Fisher Scientific, Inc.). Medium was replaced every three days.

RNA interference (RNAi). Small interfering RNA (siRNA) gene expression knockdown studies were performed according to the manufacturer's protocol. Each 27 mer RNAi duplex was transfected into cells using Lipofectamine ${ }^{\mathrm{TM}} 2000$ transfection reagent (Thermo Fisher Scientific, Inc.) according to the manufacturer's protocol. siRNA was synthesized (Guangzhou RiboBio Co., Ltd., Guangzhou, China) using the following sequences: Snail, 5'-CCACAGAAAUGGCCAUGGGAAGGC CUC-3'; and negative control, 5'-UCACAAGGGAGAGAA AGAGAGGAAGGA-3'.

Cell cytotoxicity assay. The cells were seeded into 96-well culture plates and cultured at $37^{\circ} \mathrm{C}$ for $24 \mathrm{~h}$ to attach. Subsequently, various doses $(0,2,4,6,8,10,12,14$ or $16 \mu \mathrm{mol} / \mathrm{l})$ of cisplatin (Sellack Chemicals, Houston, TX, USA) were used to treat cells as indicated and cultured at $37^{\circ} \mathrm{C}$ for $24 \mathrm{~h}$. The cells in each well containing $100 \mu \mathrm{l}$ medium were incubated with $10 \mu \mathrm{l}$ cell counting kit- 8 reagent (Beyotime Institute of Biotechnology, Haimen, China) at $37^{\circ} \mathrm{C}$ for $2 \mathrm{~h}$. The optical density of each well was subsequently measured at $450 \mathrm{~nm}$ using a microplate reader (Thermo Fisher Scientific, Inc.).

Reverse transcription-quantitative polymerase chain reaction. Total RNA was isolated by the RNeasy Plus Mini kit (Qiagen China Co., Ltd, Shanghai, China). The concentration and purity of RNA was determined by an ND-1000 spectrophotometer (NanoDrop Technologies; Thermo Fisher Scientific, Inc.). Reverse transcription was performed using the TaqMan Reverse Transcription Reagents (Applied Biosystems; Thermo Fisher Scientific, Inc.). RT-qPCR was subsequently performed using an ABI 7900 HT Fast Real-Time PCR system (Applied Biosystems; Thermo Fisher Scientific, Inc.) in the presence of SYBR-Green PCR Master Mix (Applied Biosystems; Thermo Fisher Scientific, Inc.). The gene-specific primers used are
Table I. Primer sequences used for quantitative polymerase chain reaction.

\begin{tabular}{lc}
\hline Gene & \multicolumn{2}{c}{ Primer sequence } \\
\hline Actin & \\
Forward & 5'-CACCCAGCACAATGAAGATCAAGAT-3' \\
Reverse & 5'-CCAGTTTTTAAATCCTGAGTCAAGC-3' \\
Snail & \\
Forward & 5'-TTACCTTCCAGCAGCCCTACGA-3' \\
Rerverse & 5'-GAGCCTTTCCCACTGTCCTCAT-3' \\
Slug & \\
Forward & 5'-TCCTGGTCAAGAAGCATTTCA-3' \\
Reverse & 5'-CGCCCCAAAGATGAGGAGTAT-3' \\
Zeb1 & \\
Forward & 5'-GCAGTCTGGGTGTAATCGTAAAT-3' \\
Reverse & 5'-TTGCCGTATCTGTGGTCGTG-3' \\
Zeb2 & \\
Forward & 5'-TCCCTTCTGCGACATAAATACG-3' \\
Reverse & 5'-TGTGATTCATGTGCTGCGAGTA-3' \\
\hline
\end{tabular}

Zeb, zinc finger E-box binding homeobox.

listed in Table I. Target sequences were amplified at $95^{\circ} \mathrm{C}$ for $10 \mathrm{~min}$, followed by 40 cycles at $95^{\circ} \mathrm{C}$ for $15 \mathrm{sec}$ and $60^{\circ} \mathrm{C}$ for $1 \mathrm{~min}$. $\beta$-actin was used as an endogenous normalization control. All assays were performed in triplicate. The fold change in mRNA expression was determined according to the method of $2^{\Delta \Delta \mathrm{Cq}}(16)$.

Immunocytofluorescence staining. The cells were seeded on square coverslips in six-well plates for $24 \mathrm{~h}$ to allow them to attach. Subsequently, the cells were fixed, permeated and blocked using the Immunol Fluorence Staining kit (Beyotime Institute of Biotechnology). The cells were then incubated with anti-E-cadherin antibody (diluted at 1:100; 701134; Thermo Fisher Scientific, Inc.), anti-N-cadherin antibody (diluted at 1:200; PA5-19486; Thermo Fisher Scientific, Inc.) and anti-vimentin antibody (diluted at 1:200; PA5-27231; Thermo Fisher Scientific, Inc.) overnight at $4^{\circ} \mathrm{C}$. Secondary antibody (diluted at 1:200; ab150077; Abcam, Cambridge, MA, USA) was applied for $1 \mathrm{~h}$ at room temperature. The cells were counterstained with DAPI and washed with PBS following each step of the staining procedure. Coverslips were mounted using Anti-fade Fluorescence Mounting Medium (Beyotime Institute of Biotechnology). The long and short axes of cells were measured using the Zeiss LSM Image Examiner software (version 4.2.0.121; Carl Zeiss AG, Oberkochen, Germany), and the long/short axis ratio was determined by counting 100 cells per experiment.

Western blotting. Cell lysates were extracted using radioimmunoprecipitation assay lysis buffer containing protease inhibitor cocktail (Sigma-Aldrich; EMD Millipore, Billerica, MA, USA). Protein concentrations were determined using the bicinchoninic acid method (Sigma-Aldrich; EMD Millipore). Cell lysates containing $40 \mu \mathrm{g}$ protein were loaded 
and separated on $10 \%$ SDS-PAGE gels and subsequently transferred to polyvinylidene fluoride membranes (Thermo Fisher Scientific, Inc.). The membranes were blocked in Tris Buffered Saline with 5\% (w/v) skimmed milk and $0.05 \%$ Tween 20 (Thermo Fisher Scientific, Inc.) for $1 \mathrm{~h}$ at $37^{\circ} \mathrm{C}$. Primary antibodies were incubated overnight at $4^{\circ} \mathrm{C}$. The primary antibodies and mouse monoclonal anti- $\beta$-actin were purchased from Abcam (anti-Snail antibody; ab180714; diluted at 1:1,000; anti-Slug antibody; ab27568; diluted at 1:1,000; anti-N-cadherin antibody; PA5-19486; diluted at 1:1,000; anti- $\beta$-actin antibody; ab8226; diluted at $1: 2,000)$ The $\mathrm{pH} 2 \mathrm{AX}$ antibody (MBS837487; diluted at 1:2,000) was purchased from MyBioSource, Inc. (San Diego, CA, USA). The membranes were washed and incubated with secondary antibody (ab6721; Abcam) at 1:5,000 dilution for $2 \mathrm{~h}$ at room temperature. The membranes were washed again and developed using enhanced chemiluminescence substrate (Sigma-Aldrich; EMD Millipore). Quantitative analysis was performed using QuantiOne imaging software (Bio-Rad Laboratories, Inc., Hercules, CA, USA).

Wound healing assay. A total of $5 \times 10^{5}$ cells were seeded into 6-well plates and cultured overnight at $37^{\circ} \mathrm{C}$ to attach. When adherent cells reached $\sim 90 \%$ confluence, a scratch was made using a $200-\mu 1$ pipette tip. The cells were washed three times and further incubated at $37^{\circ} \mathrm{C}$ for $24 \mathrm{~h}$. The migration was observed and recorded under a phase contrast microscope (Nikon Eclipse TE2000-U; Nikon Corporation, Tokyo, Japan).

Transwell assay. Matrigel-coated Transwell invasion assay plates (Corning Inc., Corning, NY, USA) were used for this assay. Cells were placed in the upper chamber $\left(1 \times 10^{5}\right.$ cells/well $)$ in DMEM medium with $0.1 \%$ FBS. The lower chambers were filled with DMEM medium with $10 \%$ FBS. Following culturing for $24 \mathrm{~h}$ at $37^{\circ} \mathrm{C}$, the inserts were removed and the inner side was wiped with cotton swabs. The filters were stained with Harris's hematoxylin solution (Sigma-Aldrich; EMD Millipore) for $20 \mathrm{~min}$ and peeled off following washing three times. The migrated cells were counted by a light microscope (Nikon Eclipse TE2000-U).

Animals and transplantation assay. For the in vivo assay, male NOD/SCID mice ( $\mathrm{n}=14$; 6-week-old; 18-23 g; SPF) were purchased from and maintained maintained (humidity, 50-60\%; temperature, $18-22^{\circ} \mathrm{C}$; light cycle, $10-14 \mathrm{~h}$ a day) at the Wuhan University Center for Animal Experiment (Wuhan, China). The care and use of animals was reviewed and approved by the Institutional Animal Care and Use Committee (approval number, 2011006). A total of $5 \times 10^{6}$ cells were subcutaneously injected into 2 mice, and the xenografts were obtained following two weeks of growth, and the 2 mice were sacrificed by $\mathrm{CO}_{2}$. The tumor xenografts were divided into small pieces of $\sim 5 \mathrm{~mm}^{3}$ and transplanted subcutaneously. The mice were divided into a cisplatin treated group (peritoneal injection of $5 \mathrm{mg} / \mathrm{kg}$ cisplatin once a week; $\mathrm{n}=6$ ) and the control group (receiving the same amount of saline once a week; $n=6$ ). Following 4 weeks of rearing, the mice were sacrificed by $\mathrm{CO}_{2}$. Tumor samples and lung tissues were obtained and usde for subsequent immunohistochemistry experiments.
Immunohistochemistry. Tissues were fixed in $10 \%$ neutral-buffered formalin, processed and embedded in paraffin. Tissue sections were deparaffinized and rehydrated in an ethanol series. Sections were blocked for nonspecific binding with $1 \%$ normal serum (Thermo Fisher Scientific, Inc.) and incubated with the primary anti-Snail (ab53519; diluted at 1:500; Abcam) and anti-N cadherin (PA5-19486; diluted at 1:300; Thermo Fisher Scientific, Inc.) antibodies overnight at $4^{\circ} \mathrm{C}$. Subsequently, immunostaining was developed using 3,3'-diaminobenzidine (Vector Laboratories, Inc., Burlingame, CA, USA) followed by hematoxylin counterstaining (Sigma-Aldrich; EMD Millipore). Immunostaining was visualized using a fluorescence microscope (Eclipse 80i Fluorescence Microscope; Nikon Corporation).

Statistics analysis. Each sample was analyzed in triplicate, and experiments were repeated at least two times. The mean, standard error and P-values base on the two-sample two-tailed $t$-test were calculated with Excel 2013 software (Microsoft Corporation, Redmond, WA, USA). $\mathrm{P}<0.05$ was considered to indicate a statistically significant difference.

\section{Results}

Cisplatin treatment promotes mesenchymal-like properties in osteosarcoma. Cells were treated with $5 \mu \mathrm{M}$ of cisplatin for $24 \mathrm{~h}$ and maintained in normal conditions for 5 days to recover from the chemotherapeutic stress. The surviving cells were observed to exhibit increased resistance to cisplatin (Fig. 1A). The DNA damage marker $\mathrm{pH} 2 \mathrm{AX}$ was investigated by western blotting to observe the effect of cisplatin on the cells, and the drug was confirmed to be effective (Fig. 1B). The present study observed cell morphology changes in these cisplatin-resistant cells, in which they appeared to possess a more spindle-like shape. Although there were no differences between the two groups in terms of E-cadherin and vimentin expression, $\mathrm{N}$-cadherin expression was observed to be significantly increased in the cisplatin treated group compared with the control cells (Fig. 1C). EMT is frequently accompanied by an alteration from a rounded to spindle shape in terms of cell morphology (17). Therefore, the present study assessed the long/short axis ratio in various cells. The cells treated with cisplatin were observed to have an average ratio of $3.591 \pm 0.119$, which was increased compared with the cells of the control group (average ratio, 2.232 \pm 0.041 ; $\mathrm{P}=0.001$; Fig. 1D). In addition, the expression of epithelial and mesenchymal markers was examined by western blot analysis. The epithelial markers, E-cadherin and cytokeratin, were not detectable in either the cisplatin of the control group. Mesenchymal-marker N-cadherin was highly expressed in the cisplatin group (Fig. 1E). EMT-inducing TFs, including Snail/Slug and Zeb1/2, suppress epithelial marker expression and induce the expression of mesenchymal markers, facilitating qPCR analysis that led to the observation that these four EMT-TFs were significantly upregulated (Snail, $\mathrm{P}<0.001$; Slug, $\mathrm{P}<0.001$; Zeb1, $\mathrm{P}=0.0011$; Zeb2, $\mathrm{P}<0.001)$ in the cisplatin treated cells (Fig. 2A), among which the expression of the Snail gene exhibited the most marked increase with levels of relative mRNA expression of $35.44 \pm 2.35$. Furthermore, western blotting confirmed the upregulated expression of Snail and Slug 

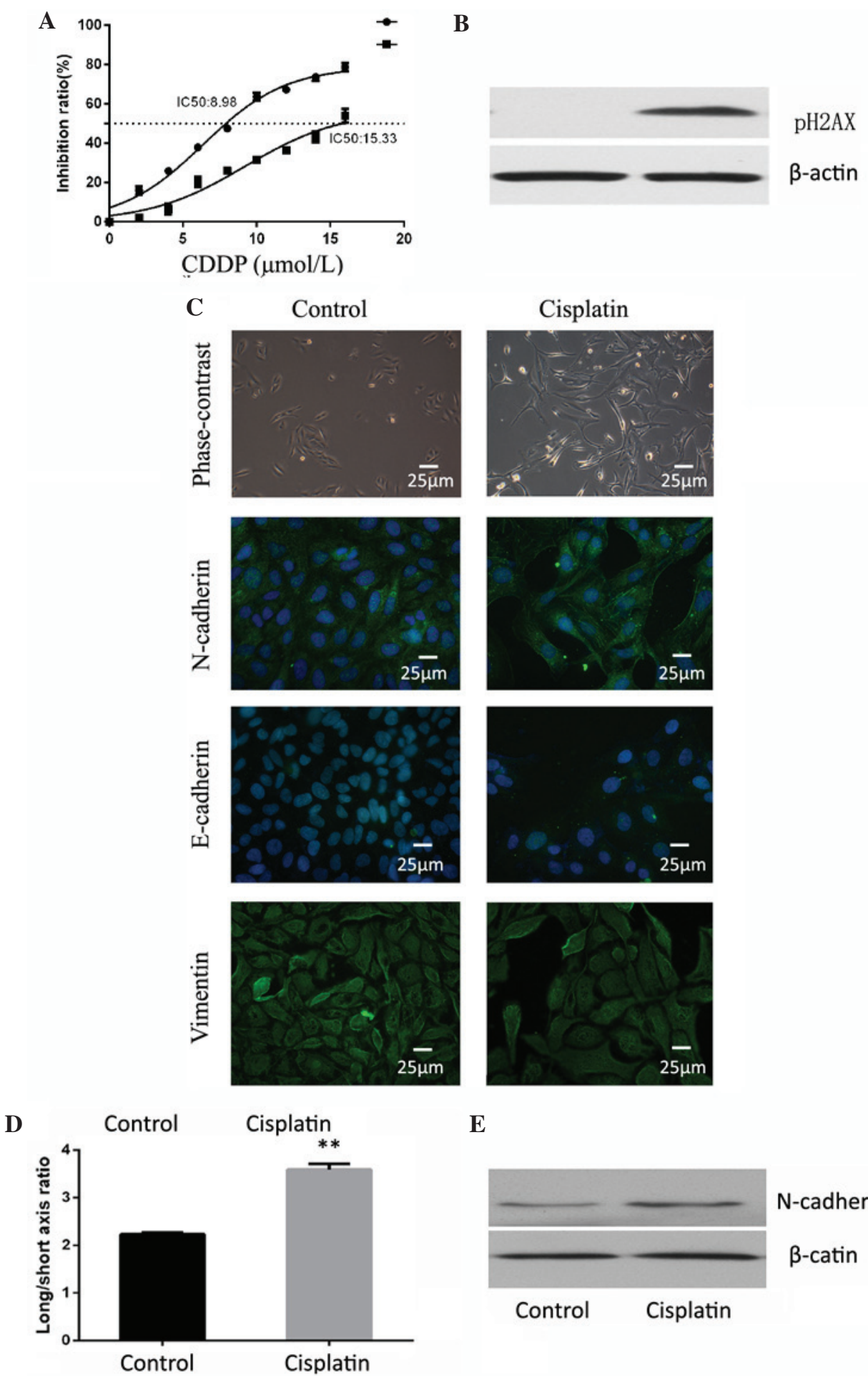

E

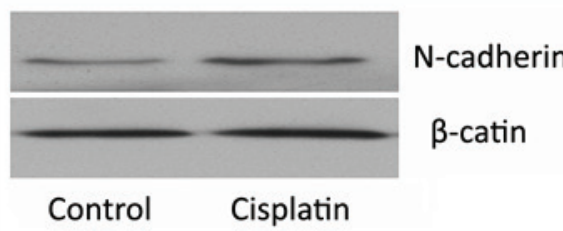

Figure 1. Cisplatin induces epithelial-mesenchymal transition in osteosarcoma. (A) Cells treated with cisplatin were observed to have increased resistance to cisplatin. (B) Cells treated with cisplatin demonstrated high expression of pH2AX and confirmed the effectiveness of cisplatin. (C) Cell shape was observed by phase contrast microscopy and immunocytofluorescence. Staining of E-cadherin, N-cadherin and vimentin for the two groups of cells was observed by fluorescence microscope (magnification, $\mathrm{x} 400$; Scale, $25 \mu \mathrm{m}$ ). Cells treated with cisplatin had higher $\mathrm{N}$-cadherin expression. (D) Cisplatin-treated group cells had a higher average ratio of long/short axis. ${ }^{* *} \mathrm{P}<0.01$. (E) Western blot analysis indicated that $\mathrm{N}$-cadherin was expressed at an increased level in the cisplatin group compared with the control.

in the cisplatin treated cells (Fig. 2B). In addition, the in vivo xenograft assay confirmed that the expression of $\mathrm{N}$-cadherin and Snail was increased following cisplatin exposure (Fig. 2C).

Cisplatin treated cells are prone to migration and invasion. Subsequently, the present study investigated the migratory and invasive capacity following cisplatin treatment. The cisplatin treated cells exhibited significantly increased cell migration compared with the control group ( $\mathrm{P}=0.001$; Fig. $3 \mathrm{~A})$. The invasive potential through the Matrigel of the cisplatin treated group was also enhanced, with an average fold increase of $1.31 \pm 0.05$ ( $\mathrm{P}=0.002$; Fig. $3 \mathrm{~B})$. To investigate the in vivo 
A
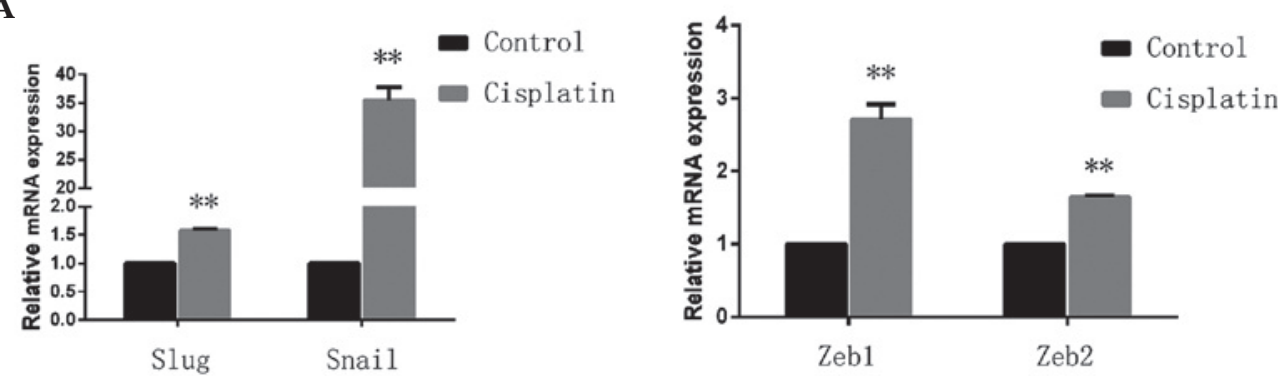

C

B

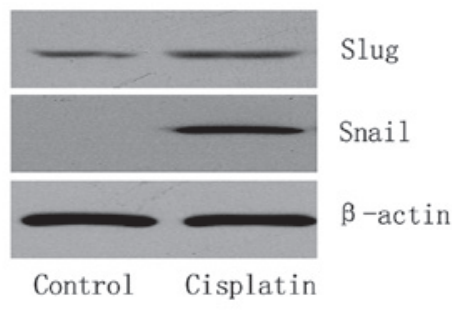

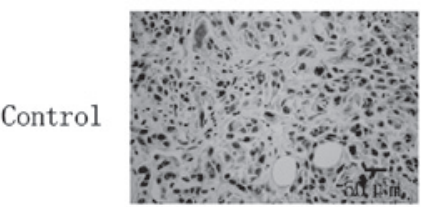

Cisplatin

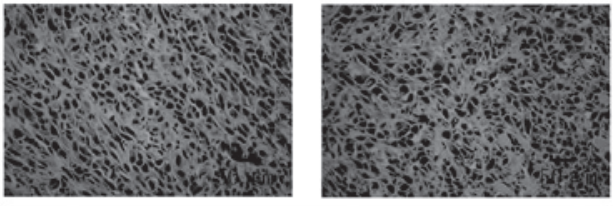

Xenograft

Figure 2. Cisplatin promotes EMT-TFs in osteosarcoma. (A) The relative expression of EMT-TFs, including Snail/Slug and Zeb1/2 were observed to be significantly upregulated in the cisplatin treated cells by quantitative polymerase chain reaction. ${ }^{* *} \mathrm{P}<0.01$. (B) Expression of Snail and Slug was analyzed by western blotting and cells treated with cisplatin demonstrated increased expression. (C) Snail and N-cadherin expression was increased following cisplatin exposure xenograft assay in vivo (magnification, x200; Scale, $50 \mu \mathrm{m}$ ). EMT, epithelial-mesenchymal transition; TF, transcription factor; Zeb, zinc finger E-box binding homeobox.

A

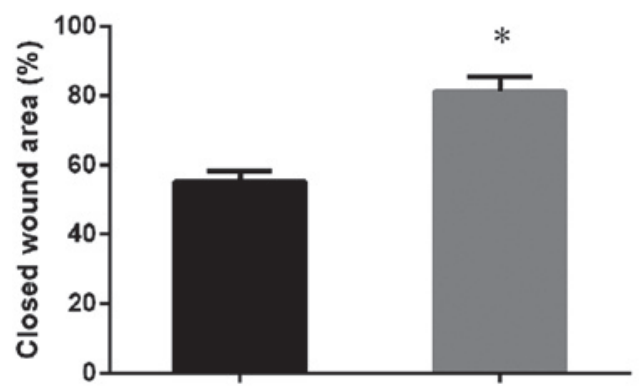

Control

Cisplatin
B

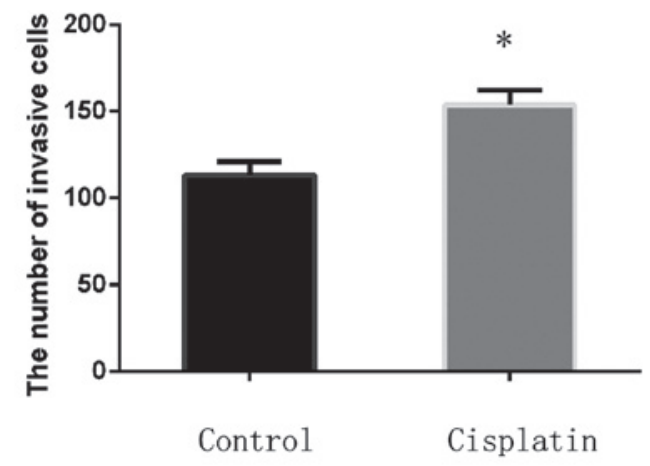

C

Xenograft pulmonary metastasis

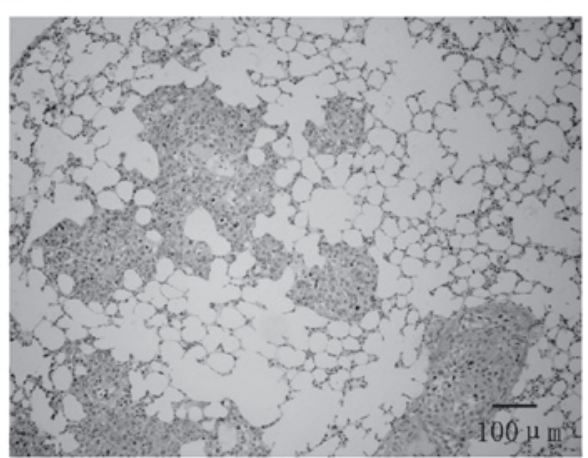

Control

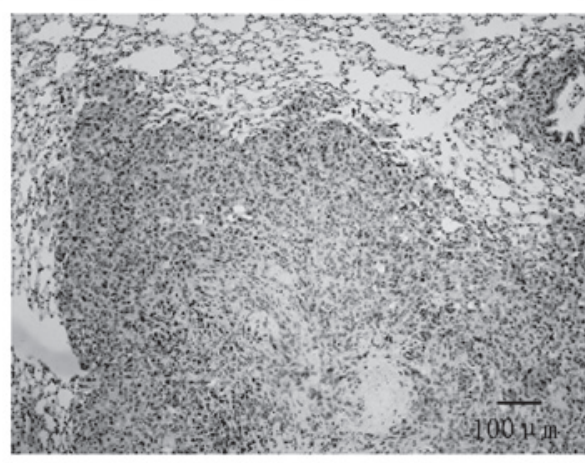

Cisplatin

Figure 3. Cisplatin promotes migratory and invasive capacity in osteosarcoma. (A) Migratory capacity was analyzed by wound healing assay and (B) invasive capacity was analyzed by Transwell assay. The cisplatin treated cells exhibited significantly increased cell migration and invasion compared with the control cells. ${ }^{*} \mathrm{P}<0.05$. (C) Transplantation assay in mice revealed that cisplatin exposure promoted pulmonary metastasis and lung destruction, although the primary tumor was inhibited (magnification, x100; Scale, $100 \mu \mathrm{m}$ ). 
A

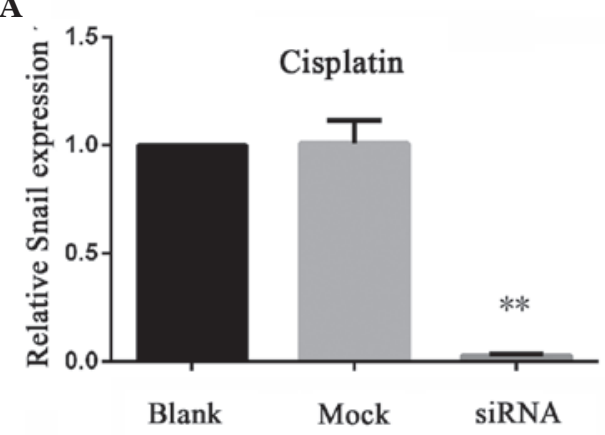

C

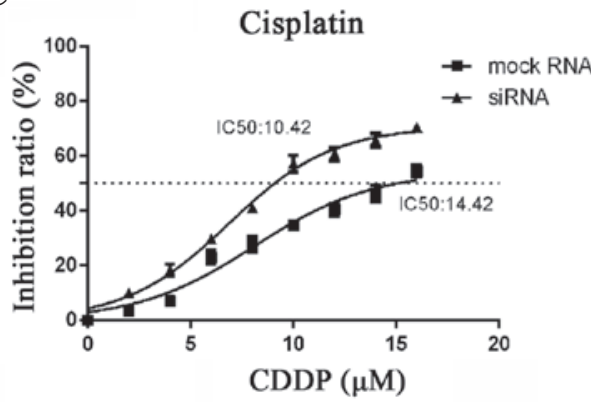

$\mathbf{E}$

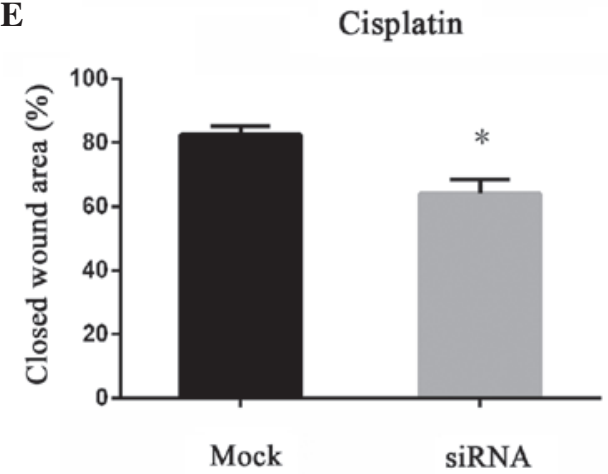

B

\section{Cisplatin}

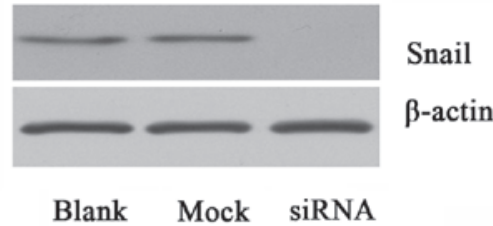

D

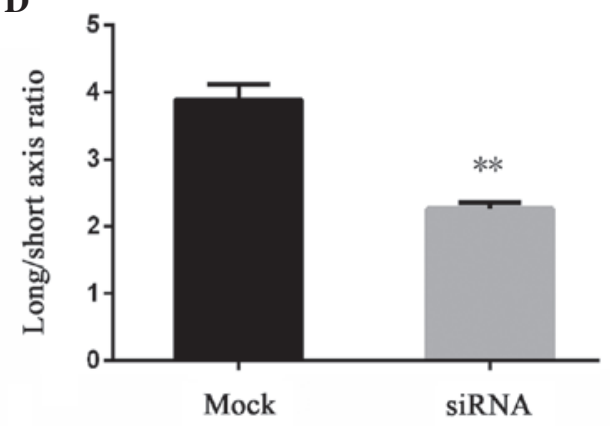

F

Cisplatin

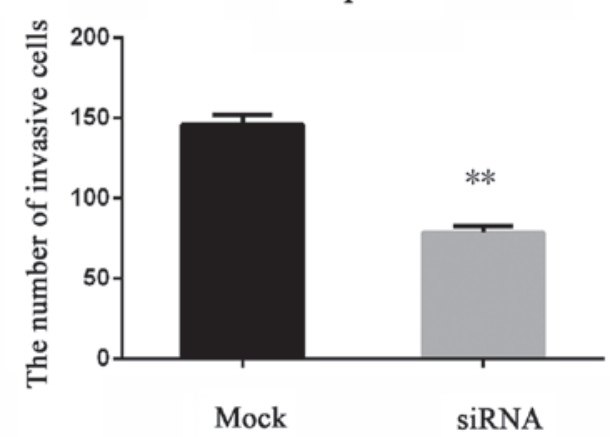

Figure 4. Snail has a significant role in cisplatin-induced epithelial-mesenchymal transition. (A) Quantitative polymerase chain reaction and (B) western blotting revealed that the expression of Snail in the siRNA group was significantly decreased compared with the blank and mock siRNA group. (C) Snail knockdown enhanced sensitivity to cisplatin. (D) Cells in the siRNA group demonstrated a decreased long/short axis ratio by phase-contrast and exhibited a reversed phenotype. Cells in the siRNA group exhibited (E) reduced migratory capacity and (F) invasive capacity. ${ }^{*} \mathrm{P}<0.05$; ${ }^{* *} \mathrm{P}<0.01$. siRNA, small interfering RNA.

metastatic capacity, the present study examined pulmonary lesions in both groups. It was observed that cisplatin exposure promoted pulmonary metastasis and induced more severe lung destruction, although the primary tumor was inhibited (Fig. 3C).

Knockdown of Snail increases cisplatin sensitivity and reverses cisplatin-induced EMT. The present study applied RNAi techniques to knockdown the expression of Snail. The efficiency of RNAi was confirmed by qPCR and western blotting (blank vs. mock group, $\mathrm{P}=0.88$; blank vs. siRNA group, $\mathrm{P}<0.001$; Fig. $4 \mathrm{~A}$ and $\mathrm{B}$ ). The $\mathrm{IC}_{50}$ for cisplatin was decreased when Snail was inhibited, which indicated that the sensitivity of osteosarcoma cells to cisplatin was enhanced (Fig. 4C). When the Snail knockdown cells were exposed to cisplatin, they exhibited a less spindle-like shape, with a decreased long/short axis ratio compared with mock cells
$(\mathrm{P}<0.001$; Fig. 4D). Furthermore, Snail inhibition blocked cisplatin-induced cell migration $(\mathrm{P}<0.001)$ and invasion $(\mathrm{P}<0.001)$ in vitro (Fig. 4E and $\mathrm{F})$.

\section{Discussion}

The introduction of chemotherapy has improved five-year survival rates of osteosarcoma (1). However, recurrence and metastasis lead to poor prognosis and are frequently associated with chemoresistance (18). EMT, a common biological process, has been reported to be associated with tumor invasiveness and migration in breast, skin and lung cancer (19-21). A previous study by the present authors reported that the concept of EMT involvement in invasiveness and migration also applies to osteosarcoma (15). In the present study, osteosarcoma cells were treated with a sublethal dose of cisplatin, and any surviving cells presented with enhanced 


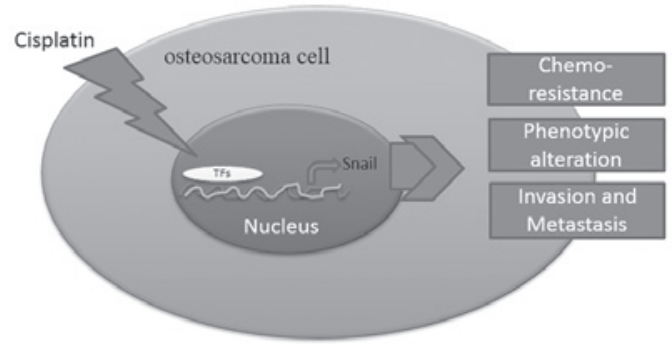

Figure 5. Schematic diagram of mechanism in cisplatin-induced epithelial-mesenchymal transition. Cisplatin stimulates the expression of Snail through transcriptional activation. Subsequently, the upregulated Snail leads to phenotypic alteration, resistance to chemotherapy and increases the capability of invasion and metastasis. TF, transcription factor.

mesenchymal-like characteristics. These cells were observed to be more resistant to cisplatin treatment, as shown by cell cytotoxicity assay. In addition, the cells demonstrated an increased expression of mesenchymal markers and an increased long/short axis ratio compared with control cells, which indicated a mesenchymal phenotype. Furthermore, the cells exhibited increased expression of EMT-inducing TFs, including Snail, Slug and Zeb1/2, which are critical in the process of EMT. The cells were also more likely to invade and migrate in vitro. In addition, xenografts treated with cisplatin demonstrated increased levels of EMT-TFs compared with those injected with saline. As the treatment time was short, it may be considered that cells with high expression of EMT-TFs, particularly Snail, demonstrated resistance to cisplatin and survived drug treatment.

In addition, to elucidate the underlying mechanisms involved in cisplatin-induced EMT, cells were transfected with siRNA targeting Snail, which was observed to have the most marked increase following cisplatin exposure in the present study. Following transfection, it was observed that the expression of Snail in the cisplatin group was decreased, while the mock cells remained unchanged. The results of the present study confirmed that the siRNA was successfully constructed and transfected into cells. Snail is a zinc-finger transcriptional repressor, which is critical to numerous biological processes, particularly in EMT $(22,23)$. A number of studies have demonstrated that Snail is able to suppress the expression of epithelial genes, primarily E-cadherin, and activate the expression of mesenchymal proteins, including $\mathrm{N}$-cadherin and fibronectin (17,24,25). In the present study, cells of the cisplatin group demonstrated increased expression of N-cadherin and Snail, and exhibited a mesenchymal phenotype. When Snail was silenced, the cells reverted to an epithelial-like phenotype. As osteosarcoma is a mesenchymal-derived tumor, the expression of E-cadherin, an epithelial gene, was low in both groups.

Snail participates in the process of EMT; in addition, recent studies have proven that Snail is involved in chemoresistance to numerous chemotherapeutic reagents (24). Hsu et al (26) reported that the expression of Snail determined the resistance to cisplatin in head and neck squamous cell carcinoma and non-small cell lung carcinoma cells and Zhang et al (27) discovered that Snail conferred resistance to 5 -fluorouracil in breast cancer cells. Similarly, in the present study, it was observed that cisplatin induced the expression of Snail in osteosarcoma cells. When Snail was suppressed, the cells became more sensitive to cisplatin. These observations appeared to indicate that Snail was involved in resistance to cisplatin in osteosarcoma. The schematic diagram in Fig. 5 shows that cisplatin promoted the binding of TFs with Snail promoter and induced its expression. Snail subsequently induced EMT leading to resistance to chemotherapy, phenotypic alteration and an increased capability of invasion and metastasis (Fig. 5).

Kudo-Saito et al (28) observed that knockdown of Snail halted tumor metastasis in melanoma. Accordingly, it was observed that the knockdown of Snail may suppress the process of EMT and inhibited the invasion and metastasis of osteosarcoma cells. Cancer stem cells are subpopulations in tumors that possess self-renewing capabilities (29). For example, cells with a cluster of differentiation (CD) $44^{\text {high }} / \mathrm{CD} 24^{\text {low }}$ phenotype were regarded as possessing stem cell traits in breast cancer (30). Mani et al (31) reported that $\mathrm{CD} 44^{\text {high }} / \mathrm{CD} 24^{\text {low }}$ cells demonstrated a decrease in E-cadherin expression and elevations in Snail and vimentin expression. Furthermore, in human mammary epithelial cells, Snail-induced EMT cells increased the capability of mammosphere-forming by 30 -fold compared with the control group (32). The pluripotent capability of stem cells was maintained in part by homeobox protein Nanog, octamer-binding transcription factor 4 (Oct4) and sex-determining region Y-box 2 (Sox2) (32,33). In a similar study of lung cancer, Wang et al (34) used A549/CDDP, a cisplatin resistance cell line, and observed that A549/CDDP cells exhibited increased expression levels of Nanog, Oct4 and Sox2. A549/CDDP cells also demonstrated increased capacities of migration and invasiveness and a higher expression of Snail. The knockdown of Snail caused a decline in migration and invasiveness in A549/CDDP cells, and the phosphoinositide 3-kinase (PI3K)/Akt signaling pathway was reported to be involved in this process (35). As well as PI3K/Akt, Snail was also able to activate the mitogen-activated protein kinase survival signaling pathways, and be activated by nuclear factor $(\mathrm{NF})-\kappa \mathrm{B}(35,36)$. Therapies with nitric oxide, the proteasome inhibitor NPI-0052 targeting NF- $\kappa \mathrm{B}$ and Snail were proven to be effective in prostate cancer and B-non-Hodgkin's lymphoma (37).

In conclusion, the EMT concept may be applied to osteosarcoma, which is a mesenchymal-derived tumor. Targeting Snail and other EMT-TFs may assist with preventing metastasis of osteosarcoma patients. Chemical inhibitors targeting Snail and other EMT-TFs have been reported to be effective in cell lines and animal experiments (38-41). As well as U2OS, future studies must investigate other cell lines in osteosarcoma to increase credibility. Additional studies and clinical trials are required to evaluate the efficacy of targeting EMT-TFs in the prevention of cancer recurrence, metastasis and chemoresistance.

\section{Acknowledgments}

The present study was supported by grants from the National Natural Science Foundation of China (grant no., 81341078) and the Science and Technology Foundation of Wuhan (grant no., 2014062801011264). 


\section{References}

1. Ottaviani G and Jaffe N: The epidemiology of osteosarcoma. Cancer Treat Res 152: 3-13, 2009.

2. Mirabello L, Troisi RJ and Savage SA: Osteosarcoma incidence and survival rates from 1973 to 2004: Data from the Surveillance, Epidemiology, and End Results Program. Cancer 115: 1531-1543, 2009.

3. Clark JC, Dass CR and Choong PF: A review of clinical and molecular prognostic factors in osteosarcoma. J Cancer Res Clin Oncol 134: 281-297, 2008.

4. Chen Y, Han F, Cao LH, Li C, Wang JW, Li Q, Zheng W, Guo ZX, Li AH and Zhou JH: Dose-response relationship in cisplatin-treated breast cancer xenografts monitored with dynamic contrast-enhanced ultrasound. BMC Cancer 15: 136, 2015.

5. Ebrahimi Shahmabadi H, Movahedi F, Koohi Moftakhari Esfahani M, Alavi SE, Eslamifar A, Mohammadi Anaraki G and Akbarzadeh A: Efficacy of Cisplatin-loaded polybutyl cyanoacrylate nanoparticles on the glioblastoma. Tumour Biol 35: 4799-4806, 2014.

6. Pirker R: Adjuvant chemotherapy in patients with completely resected non-small cell lung cancer. Transl Lung Cancer Res 3: 305-310, 2014.

7. Hjelle LV, Gundersen PO, Oldenburg J, Brydøy M, Tandstad T, Wilsgaard T, Fosså SD, Bremnes RM and Haugnes HS: Long-term platinum retention after platinum-based chemotherapy in testicular cancer survivors: A 20-year follow-up study. Anticancer Res 35: 1619-1625, 2015.

8. Woźniak K and Błasiak J: Recognition and repair of DNA-cisplatin adducts. Acta Biochim Pol 49: 583-596, 2002.

9. Haddox CL, Han G, Anijar L, Binitie O, Letson GD, Bui MM and Reed DR: Osteosarcoma in pediatric patients and young adults: A single institution retrospective review of presentation, therapy and outcome. Sarcoma 2014: 402509, 2014.

10. Jeanes A, Gottardi CJ and Yap AS: Cadherins and cancer: How does cadherin dysfunction promote tumor progression? Oncogene 27: 6920-6929, 2008.

11. Han SP and Yap AS: The cytoskeleton and classical cadherin adhesions. Subcell Biochem 60: 111-135, 2012.

12. Azmi AS: Unveiling the role of nuclear transport in epithelial-to-mesenchymal transition. Curr Cancer Drug Targets 13: 906-914, 2013.

13. Patel IS, Madan P, Getsios S, Bertrand MA and MacCalman CD: Cadherin switching in ovarian cancer progression. Int $\mathrm{J}$ Cancer 106: 172-177, 2003.

14. Lim J and Thiery JP: Epithelial-mesenchymal transitions: Insights from development. Development 139: 3471-3486, 2012.

15. Yu L, Liu S, Guo W, Zhang C, Zhang B, Yan H and Wu Z: hTERT promoter activity identifies osteosarcoma cells with increased EMT characteristics. Oncol Lett 7: 239-244, 2014.

16. Livak and Schmittgen: Analysis of relative gene expression data using real-time quantitative PCR and the $2-\Delta \Delta \mathrm{Ct}$ method. Methods 25: 402-408, 2001.

17. Wang Y, Shi J, Chai K, Ying X and Zhou BP: The Role of Snail in EMT and Tumorigenesis. Curr Cancer Drug Targets 13: 963-972, 2013.

18. Bruheim S, Xi Y, Ju J and Fodstad O: Gene expression profiles classify human osteosarcoma xenografts according to sensitivity to doxorubicin, cisplatin, and ifosfamide. Clin Cancer Res 15: 7161-7169, 2009

19. Zhou F, Liu H, Zhang X, Shen Y, Zheng D, Zhang A, Lai Y and Li H: Proline-rich protein 11 regulates epithelial-to-mesenchymal transition to promote breast cancer cell invasion. Int J Clin Exp Pathol 7: 8692-8699, 2014.

20. Chen CH, Lin DS, Cheng CW, Lin CJ, Lo YK, Yen CC, Lee AY and Hsiao CD: Cdc6 cooperates with c-Myc to promote genome instability and epithelial to mesenchymal transition EMT in zebrafish. Oncotarget 5: 6300-6311, 2014.

21. Grelet S, Andries V, Polette M, Gilles C, Staes K, Martin AP, Kileztky C, Terryn C, Dalstein V, Cheng CW, et al: The human NANOS3 gene contributes to lung tumour invasion by inducing epithelial-mesenchymal transition. J Pathol 237: 25-37, 2015.

22. Barrallo-Gimeno A and Nieto MA: The Snail genes as inducers of cell movement and survival: Implications in development and cancer. Development 132: 3151-3161, 2005.
23. Peinado H, Olmeda D and Cano A: Snail, Zeb and bHLH factors in tumour progression: An alliance against the epithelial phenotype? Nat Rev Cancer 7: 415-428, 2007.

24. Kim YS, Yi BR, Kim NH and Choi KC: Role of the epithelial-mesenchymal transition and its effects on embryonic stem cells. Exp Mol Med 46: e108, 2014.

25. Kaufhold S and Bonavida B: Central role of Snail1 in the regulation of EMT and resistance in cancer: A target for therapeutic intervention. J Exp Clin Cancer Res 33: 62, 2014.

26. Hsu DS, Lan HY, Huang CH, Tai SK, Chang SY, Tsai TL, Chang CC, Tzeng CH, Wu KJ, Kao JY and Yang MH: Regulation of excision repair cross-complementation group 1 by Snail contributes to cisplatin resistance in head and neck cancer. Clin Cancer Res 16: 4561-4571, 2010.

27. Zhang W, Feng M, Zheng G, Chen Y, Wang X, Pen B, Yin J, $\mathrm{Yu} \mathrm{Y}$ and $\mathrm{He} \mathrm{Z}$ : Chemoresistance to 5-fluorouracil induces epithelial-mesenchymal transition via up-regulation of Snail in MCF7 human breast cancer cells. Biochem Biophys Res Commun 417: 679-685, 2012.

28. Kudo-Saito C, Shirako H, Takeuchi T and Kawakami Y: Cancer metastasis is accelerated through immunosuppression during Snail-induced EMT of cancer cells. Cancer Cell 15: 195-206, 2009.

29. Gupta PB, Chaffer CL and Weinberg RA: Cancer stem cells: Mirage or reality? Nat Med 15: 1010-1012, 2009.

30. Al-Hajj M, Wicha MS, Benito-Hernandez A, Morrison SJ and Clarke MF: Prospective identification of tumorigenic breast cancer cells. Proc Natl Acad Sci USA 100: 3983-3988, 2003.

31. Mani SA, Guo W, Liao MJ, Eaton EN, Ayyanan A, Zhou AY, Brooks M, Reinhard F, Zhang CC, Shipitsin M, et al: The epithelial-mesenchymal transition generates cells with properties of stem cells. Cell 133: 704-715, 2008.

32. Takahashi K and Yamanaka S: Induction of pluripotent stem cells from mouse embryonic and adult fibroblast cultures by defined factors. Cell 126: 663-676, 2006.

33. Moon JH, Yun W, Kim J, Hyeon S, Kang PJ, Park G, Kim A, Oh S, Whang KY, Kim DW, et al: Reprogramming of mouse fibroblasts into induced pluripotent stem cells with Nanog. Biochem Biophys Res Commun 431: 444-449, 2013.

34. Wang H, Zhang G, Zhang H, Zhang F, Zhou B, Ning F, Wang HS, Cai SH and Du J: Acquisition of epithelial-mesenchymal transition phenotype and cancer stem cell-like properties in cisplatin-resistant lung cancer cells through AKT/ $\beta$-catenin/Snail signaling pathway. Eur J Pharmacol 723: 156-166, 2014

35. Vega S, Morales AV, Ocaña OH, Valdés F, Fabregat I and Nieto MA: Snail blocks the cell cycle and confers resistance to cell death. Genes Dev 18: 1131-1143, 2004.

36. Vega MI, Baritaki S, Huerta-Yepez S, Martinez-Paniagua MA and Bonavida B: A potential mechanism of rituximab-induced inhibition of tumor growth through its sensitization to tumor necrosis factor-related apoptosis-inducing ligand-expressing host cytotoxic cells. Leuk Lymphoma 52: 108-121, 2011.

37. Baritaki S, Yeung K, Palladino M, Berenson J and Bonavida B: Pivotal roles of snail inhibition and RKIP induction by the proteasome inhibitor NPI-0052 in tumor cell chemoimmunosensitization. Cancer Res 69: 8376-8385, 2009.

38. Shah P, Gau Y and Sabnis G: Histone deacetylase inhibitor entinostat reverses epithelial to mesenchymal transition of breast cancer cells by reversing the repression of E-cadherin. Breast Cancer Res Treat 143: 99-111, 2014.

39. Miller CR, Oliver KE and Farley JH: MEK1/2 inhibitors in the treatment of gynecologic malignancies. Gynecol Oncol 133: 128-137, 2014.

40. McCubrey JA, Steelman LS, Chappell WH, Abrams SL, Franklin RA, Montalto G, Cervello M, Libra M, Candido S, Malaponte G, et al: Ras/Raf/MEK/ERK and PI3K/PTEN/Akt/mTOR cascade inhibitors: How mutations can result in therapy resistance and how to overcome resistance. Oncotarget 3: 1068-1111, 2012.

41. Bruheim S, Xi Y, Ju J and Fodstad O: Gene expression profiles classify human osteosarcoma xenografts according to sensitivity to doxorubicin, cisplatin, and ifosfamide. Clin Cancer Res 15: 7161-7169, 2009. 